\title{
Deep Penetrating Nevus: A Rare Variant of Melanocytic Nevus
}

\author{
El Jouari 0 ${ }^{1 *}$, Senhaji G ${ }^{1}$, Gallouj $\mathbf{S}^{1}$, Hammas $\mathbf{N}^{2}$ and Mernissi FZ ${ }^{1}$ \\ ${ }^{1}$ Department of Dermatology, CHU Hassan II Fez, Morocco \\ ${ }^{2}$ Department of Anatomopathology, CHU Hassan II Fez, Morocco
}

*Corresponding author: Ouiame El Jouari, Department of Dermatology and Venereology, University Hospital Hassan II Fez, Morocco, Tel: 00212645768798; Email: eljouariouiame@gmail.com

\section{Case Report \\ Volume 3 Issue 3}

Received Date: October 05, 2018

Published Date: November 05, 2018

DOI: $10.23880 /$ cdoaj-16000157

\section{Abstract}

Deep penetrating nevus (DPN) is a distinctive melanocytic nevus. The most frequent clinical presentation is that of a deeply pigmented papule on the face, head, neck, trunk, or proximal extremity. Histopathologically, the DPN is wedgeshaped and contains spindles melanocytes that exhibit deep infiltration into the dermis. It can be falsely diagnosed as malignant melanoma. Simple excision of the lesion is usually curative. We report an uncommon case of congenital DPN, which should be considered in any young patient presenting with a new or changing darkly pigmented papule or nodule.

Keywords: Deep penetrating nevus; Plexiform spindle cell nevus; Melanoma

\section{Abbreviations: DPN: Deep Penetrating Nevus.}

\section{Introduction}

The deep penetrating nevus (DPN), also known as the plexiform spindle cell nevus, is a pigmented lesion that commonly arises on the head and neck in the first few decades of life [1]. DPN needs to be distinguished from malignant melanoma and also from other benign lesions as blue nevus, cellular blue nevus, spindle and epithelioid nevus, pigmented spindle cell nevus and congenital melanocytic nevus [2]. We present an uncommon case of congenital DPN, describe its clinical, dermoscopic and histopathologic features. At 24 months of decline, no recurrence was noted.

\section{Case Report}

We report a case of an 8-year-old Moroccan boy who was presented to our department with a solitary asymptomatic congenital pigmented lesion of the nose which had increasing in size. Clinical examination revealed a $10 \mathrm{~mm}$ solitary, symmetrical brown bluish nodule, firm in consistency, located on the right nostril (Figure 1). No lymph node was observed. Dermatoscopy of the lesion showed a homogenous, brown-blue and globular pattern with emergence of some hair (Figure 2). A complete surgical excision was performed. Histological examination revealed a dermo-hypodermic melanocytic proliferation mostly circumscribed to the dermis. This proliferation contains spindles melanocyte cells with abundant cytoplasm punctuated by fine grains of pigment. It was not seen cytological atypia (Figure 3). Immunohistochemical staining was positive for S-100 and HMB-45 (Figure 4). A diagnosis of intradermal melanocytic proliferation with features of DPN was made. A complete surgical excision was performed. At 24 months of decline, no recurrence was noted. 


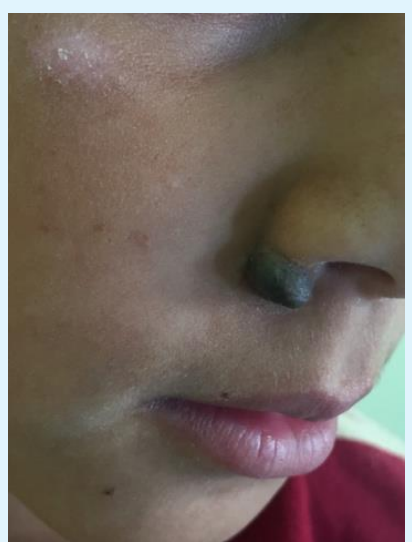

Figure 1: A $10 \mathrm{~mm}$ brown bluish nodule of the right nostril.

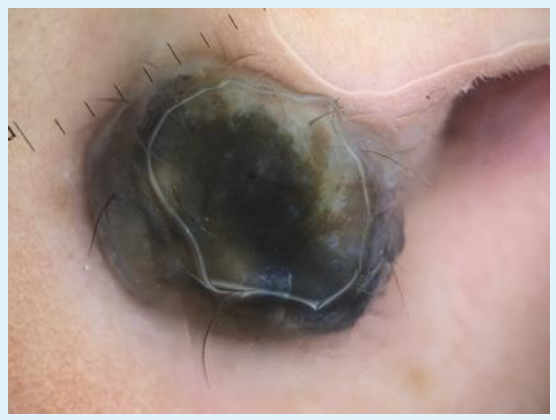

Figure 2: Dermoscopy showing a homogenous, brownblue and globular pattern with emergence of some hair.

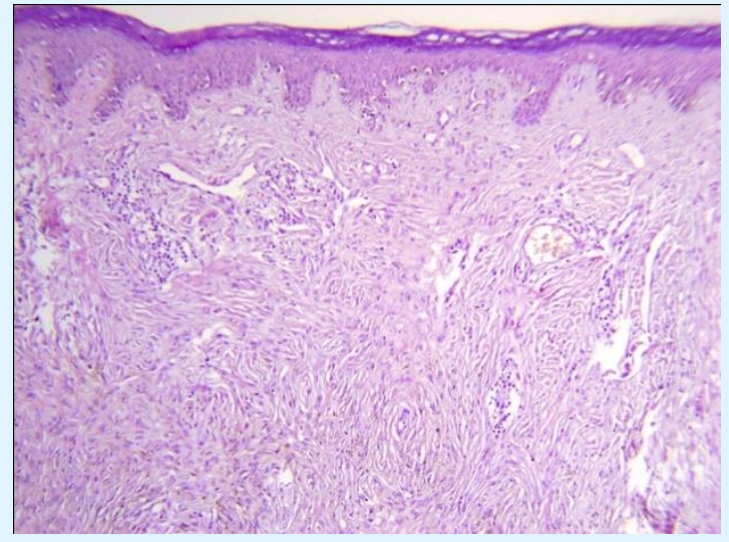

Figure 3: Deep penetrating naevi are characterized by plump fusiform melanocytes, without atypia.

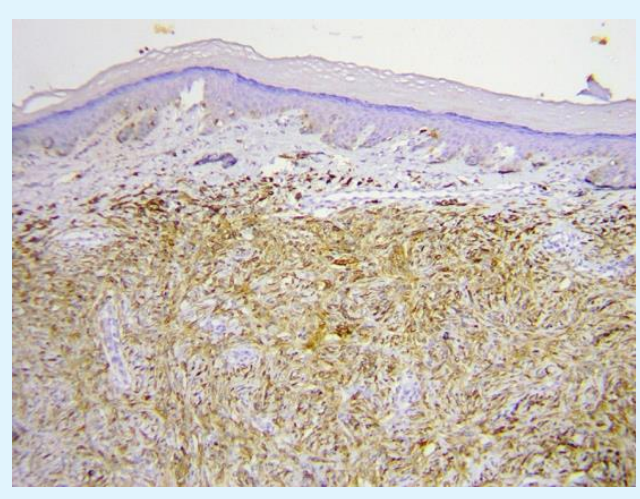

Figure 4: Immunohistochemical staining positive for S100 and HMB-45.

\section{Discussion}

The Deep penetrating nevus is a rare, benign melanocytic tumor first described by Seab, et al. in 1989 [2]. The most frequent clinical presentation is that of a deeply pigmented (brown to black) papule, less than $1 \mathrm{~cm}$ in diameter, primarily located on the face, neck, trunk, and proximal extremities [3]. Deep penetrating nevus usually affects young individuals [4]. The dermoscopic features of DPN have not been well established [1]. Some authors described asymmetrical, regular, "negative" globular pattern with underlying homogenous blue-brown pigmentation [5] and polychromatic pattern (blue, brown and white) [6].There is still controversy as to whether DPN represents a distinct clinical entity or simply a variant of blue nevus; in addition, DPN has also frequently been found in association with other nevi, thus forming a combined nevus. Recently, authors concluded that, unless DPN shared histological features with other nevi, it could be regarded as a distinct entity in most cases [7]. Histopathologically, DPN is a sharply demarcated lesion, and at low power is symmetric and wedged-shaped with a broad base that runs parallel with the epidermis and an apex that is oriented towards the subcutaneous adipose. It consists of often vertically oriented, loose fascicles and nests of spindle-shaped and epithelioid melanocytes extending into the reticular dermis in a plexiform pattern [1].In particular, unlike blue nevi, nevus cells may have a round contour, and are interspersed with scarce melanophages; moreover, compared to other benign melanocytic lesions, nevus cellularity is usually high in DPN [3]. At higher magnification, nevus cells show pleomorphic nuclei with nuclear pseudo-inclusions; mitotic activity is low or absent. Lesional cell nests tend to surround adnexal structures. When present, the 
inflammatory infiltrate is primarily composed of lymphoid elements. Unlike DPN, malignant melanoma is characterized by an asymmetrical and poorly circumscribed proliferation of atypical melanocytes with destructive growth in the dermis, frequent infiltration into the subcutis, and more pronounced cytologic atypia with frequent mitoses [8]. On immunohistochemical analysis, DPN shows positivity for both S-100 and HMB45 and negativity for keratins. Hence, immunohistochemistry is of no value in differentiating this entity from malignant melanoma [2]. DPN differential diagnosis includes blue naevus, melanocytic naevus, atypical nevus, and melanoma [4]. The earlier reports of DPN discuss is by complete excision. However, perhaps because DPN can clinically and histologically appear similar to malignant melanoma, some clinicians re-excise DPN with margins [1].

\section{Conclusion}

DPN is a benign melanocytic lesion that may be mistaken for melanoma. Consequently, the histopathologic diagnosis is crucial to differentiate both entities. It should be considered in any young patient presenting with a new or changing darkly pigmented papule or nodule.

\section{References}

1. Strazzula L, Senna MM, Yasuda M, Belazarian L (2014) The deep penetrating nevus. J Am Acad Dermatol Dec 71(6): 1234-1240.
2. Gupta A, Srilatha P S, Suvarna N, Rao L (2011) Deep penetrating nevus: A distinct variant of melanocytic nevus. Indian J Pathol Microbiol 54(1): 156-157.

3. Veronese F, Celasco M, Meli F, Zavattaro E, Ramponi A, et al. (2016) Deep penetrating nevus of the plantar surface: report of a case with dermatoscopic features. J Dtsch Dermatol Ges 14(5): 517-518.

4. Pérez OG (2009) Deep penetrating naevus. JEADV 23: 702-738.

5. Robson A, Morley-Quante M, Hempel H, McKee PH, Calonje E (2003) Deep penetrating nevus: clinicopathological study of 31 cases with further delineation of histological features allowing distinction from other pigmented benign melanocytic lesions and melanoma. Histopathology 43(6): 529537.

6. Ferrara G, Soyer HP, Malvehy J, Piccolo D, Puig S, et al. (2007) The many faces of blue nevus: a clinicopathologic study. J Cutan Pathol 34(7): 543551.

7. Barnhill RL, Cerroni L, Cook M, Elder DE, Kerl H, et al. (2010) State of the art, nomenclature, and points of consensus and controversy concerning benign melanocytic lesions: outcome of an international workshop. Adv Anat Pathol 17: 73-90.

8. Luzar B, Calonje E (2011) Deep penetrating nevus: a review. Arch Pathol Lab Med 135(3): 321-326.

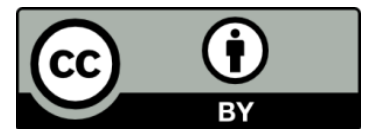

